\title{
Impact de l'association sur le niveau de nutrition azotée et la croissance du ray-grass anglais et du trèfle blanc
}

\author{
JF Soussana*, MC Arregui ** \\ INRA, station d'agronomie, 12, avenue du Brézet, F63039 Clermont-Ferrand cedex 02, France
}

(Reçu le 17 novembre 1994 ; accepté le 12 avril 1995)

\begin{abstract}
Résumé - La nutrition azotée de cultures pures et d'associations fauchées de trèfle blanc et de ray-grass anglais semées en rangs alternés a été étudiée, durant 2 repousses de printemps, avec ou sans fertilisation azotée, par la méthode des courbes de dilution de l'azote total dans la matière sèche. L'association avec le trèfle blanc a augmenté la teneur en azote total et la biomasse épigée par mètre linéaire des rangs de ray-grass. À même biomasse, la teneur en azote du ray-grass associé est supérieure à celle mesurée en culture pure. Le niveau moyen de nutrition azotée du ray-grass augmente généralement de manière hautement significative en culture associée, mais cet effet est toutefois plus faible après une fertilisation azotée. Un sous-essai, où les parties aériennes et les racines du trèfle et du ray-grass étaient séparées, ou non, par des cloisons verticales, a indiqué, à la dernière repousse, une augmentation significative du niveau de nutrition azotée du ray-grass en cas d'interférence racinaire avec le trèfle. Dans le cas du trèfle blanc, la diminution de la teneur en azote total des feuilles au cours de le repousse s'explique, en partie, par la réduction du rapport pondéral (limbes/pétioles). Par ailleurs, la teneur en azote des limbes de trèfle diminue selon une loi puissance en fonction de la biomasse foliaire totale du peuplement. La culture en association n'a pas d'effet significatif sur les teneurs en azote des limbes de trèfle, lorsque celles-ci sont comparées à même biomasse foliaire totale. Néanmoins, le taux de trèfle dans l'association varie en raison inverse du niveau de nutrition azotée du ray-grass.
\end{abstract}

courbe de dilution / teneur en azote / compétition / Lolium perenne $L=$ ray-grass / Trifolium repens $L=$ trèfle blanc

Summary - Effect of mixed cropping on the nitrogen nutrition and growth of perennial ryegrass and white clover. The nitrogen nutrition of cut white clover and perennial ryegrass monocultures and mixtures sown in adjacent rows was studied during 2 spring regrowths, with and without a nitrogenous fertilizer supply, using the nitrogen dilution curve method. Ryegrass shoot biomass per row and shoot nitrogen concentration increased in the mixture compared with the monoculture. For a similar ryegrass shoot biomass, the nitrogen concentration was higher in the mixed than in the pure ryegrass. Perennial ryegrass shoot nitrogen levels increased significantly in the mixture, but this effect was weaker after nitrogen fertilization. A sub-trial where clover and grass shoots and roots were separated by vertical partitions, or mixed, indicated a significant increase in the nitrogen nutrition level of ryegrass at the final cut, due to root interaction between both species. The decline in leaf nitrogen concentration of white clover during regrowth was partly

\footnotetext{
* Correspondance et tirés à part

** Adresse actuelle : Universidad del Litoral, Esperanza, Argentine
} 
due to a decline in the lamina/petiole ratio. Furthermore, when the sward total leaf biomass increased, clover lamina nitrogen concentration declined according to a power function. At a given total leaf biomass, clover lamina nitrogen concentration was not significantly affected by association with ryegrass. Nevertheless, the clover content of the mixed sward was negatively correlated with ryegrass nitrogen nutrition level.

dilution curve / nitrogen concentration / competition / Lolium perenne $L$ / Trifolium repens $L$

\section{INTRODUCTION}

Dans une association fauchée graminée-légumineuse, les 2 espèces ont la capacité d'assimiler l'azote minéral du sol, mais seule la légumineuse a la capacité de fixer de manière symbiotique l'azote atmosphérique. Une fraction de l'azote fixé est généralement libérée dans le sol après la sénescence et la décomposition des tissus végétaux de la légumineuse, ou encore par l'exsudation de composés azotés dans la rhizosphère (Harris, 1987). L'enrichissement du sol qui en résulte affecte, en retour, les compétitions racinaires intra- et interspécifiques pour l'azote (Haynes, 1980 ; Harris, 1987). Au total, ces interférences (Harper, 1977) pour l'azote peuvent conduire à des modifications des niveaux de nutrition azotée chez les 2 espèces, ainsi que du rapport assimilation-fixation chez la légumineuse.

L'effet global bénéfique de la présence d'une légumineuse sur la nutrition azotée de la graminée a été signalé par de nombreux auteurs (Birch et Dougall, 1967 ; Charles, 1982) sur la base d'une augmentation de la teneur en azote total foliaire de la graminée. Pour le couple trèfle blanc/ray-grass anglais, la culture en association accroit la teneur en azote de la graminée (Broadbent et al, 1982 ; Arnaud et Niqueux, 1986). Toutefois, Cruz et Lemaire (1986b) ont montré que la simple détermination de la teneur en azote pouvait conduire à une surestimation de l'effet améliorateur de la légumineuse sur la nutrition azotée de la graminée. La teneur en azote total des parties récoltées diminue en effet au cours de la repousse des peuplements prairiaux (Salette et Lemaire, 1981; Lemaire et Salette, 1984). La teneur plus forte de la graminée associée peut donc résulter, non d'une amélioration de son niveau de nutrition azotée, mais de son moindre degré de croissance, comme dans le cas du dactyle associé à la luzerne (Cruz et Lemaire, 1986b).

En levant partiellement l'inhibition de la fixation symbiotique par l'azote minéral du sol (Streeter, 1988), les prélèvements d'azote du ray-grass ont pour effet d'accroître le taux de fixation du trèfle
(Brophy et al, 1987 ; Böller et Nösberger, 1987). La légumineuse est alors plus étroitement dépendante des processus symbiotiques pour sa nutrition azotée. Or, la fixation symbiotique du trèfle dépend également fortement de sa biomasse foliaire (Gückert et al, 1983 ; Mariott, 1988) et de l'éclairement reçu dans le couvert (Faurie et Soussana, 1993). La fixation de l'azote pourrait donc diminuer en cas d'ombrage des feuilles par la graminée associée, ce qui pourrait expliquer l'effet négatif de l'association sur la teneur en azote épigée du trèfle (Broadbent et al, 1982) et rendre compte, éventuellement, de la fréquente régression du trèfle blanc dans les associations recevant une fertilisation azotée (Frame et Newbould, 1986).

L'objectif de notre travail a donc été d'apporter des éléments de réponse aux questions suivantes:

- l'association avec le trèfle améliore-t-elle le niveau de nutrition azotée du ray-grass ?

- l'association avec le ray-grass affecte-t-elle la nutrition azotée du trèfle?

- la fréquente régression du trèfle dans l'association après une fertilisation azotée peut-elle s'expliquer par des modifications du niveau de nutrition azotée des 2 partenaires de l'association?

Cruz et Lemaire (1986a, b) ont analysé l'effet propre de la compétition interspécifique en comparant des luzernes et des dactyles cultivés à double écartement avec des dactyles associés à la luzerne à simple écartement. Un tel dispositif additif n'était pas applicable au trèfle blanc, cette espèce stolonifère ayant la capacité de coloniser rapidement l'inter-rang.

La comparaison de cultures pures et associées de même écartement (expériences en substitution) permet d'analyser les effets du remplacement d'une partie de la compétition intraspécifique par une compétition interspécifique, les lignes adjacentes de la même espèce étant remplacées par celles de l'espèce associée (De Wit et al, 1966 ; Jacquard, 1968). Dans une revue récente, Sackville-Hamilton (1994) montre que les dispositifs en substitution doivent être préférés aux dispositifs additifs, si l'on cherche à com- 
parer les effets relatifs des compétitions intra- et inter-spécifiques.

Dans ce but, des cultures pures et associées de trèfle et de ray-grass de même écartement ont été comparées durant la période de croissance printanière et leur nutrition azotée a été étudiée, grâce la méthode des courbes de dilution. De plus, nous avons tenté de séparer les effets des compétitions interspécifiques racinaires et épigées, afin d'évaluer leurs poids relatifs dans les phénomènes observés.

\section{MATÉRIELS ET MÉTHODES}

\section{Dispositif expérimental}

L'essai a été implanté à Theix (altitude $890 \mathrm{~m}$, Massif central) sur un sol brun sablo-limoneux $(\mathrm{pH} \mathrm{6,2)} \mathrm{à} 4 \%$ de matière organique. Une fertilisation phospho-potassique de $150 \mathrm{~kg} \mathrm{P}_{2} \mathrm{O}_{5} \cdot$ ha $^{-1}$ et $350 \mathrm{~kg} \mathrm{~K}_{2} \mathrm{O} \cdot$ ha $^{-1}$ a été apportée avant semis puis, 1 an plus tard, au sortir de I'hiver.

Quelque 60 micro-parcelles de $8 \mathrm{~m}^{2}$ ont été semées au printemps en rangs (inter-rang de $17 \mathrm{~cm}$ ) avec un semoir de précision, à une densité d'environ 200 graines viables par mètre linéaire pour les 2 espèces. Dans le cas des associations, trèfle et raygrass ont été semés en rangs alternés. Le trèfle blanc (Trifolium repens $\mathrm{L}$ ), est de type Hollandicum (cv Grasslands Huïa) et le ray-grass anglais (Lolium perenne L) un diploïde à épiaison tardive (cv Préférence). Le dispositif expérimental comprend 5 blocs complets, croisant 2 facteurs :

- le type de culture : ray-grass pur, trèfle pur, ou association des 2 ;

- la fertilisation azotée : pas d'apport d'azote minéral $\left(\mathrm{N}_{0}, \mathrm{~N}_{00}\right) ; 100 \mathrm{~kg} \mathrm{~N} \cdot \mathrm{ha}^{-1}$ au début de la première repousse de printemps $\left(\mathrm{N}_{1}, \mathrm{~N}_{10}\right) ; 100 \mathrm{~kg} \mathrm{~N}^{\mathrm{h} h a^{-1} \text { au }}$ début de la seconde repousse de printemps $\left(N_{0}, N_{01}\right)$; $100 \mathrm{~kg} \mathrm{~N}^{\text {h ha-1 }}{ }^{-1}$ au début des 2 repousses de printemps $\left(N_{1}, N_{11}\right)$. Les notations à un indice $\left(N_{0}, N_{1}\right)$ correspondent à la première repousse ; les notations à 2 indices à la seconde $\left(\mathrm{N}_{00}, \mathrm{~N}_{01}, \mathrm{~N}_{10}, \mathrm{~N}_{11}\right)$.

Après l'année d'implantation, les coupes ont été réalisées à un rythme lent (durée de repousse d'environ $50 \mathrm{j}$ ) à une hauteur de $4 \mathrm{~cm}$ environ. Les températures moyennes des 2 périodes de repousse de printemps étudiées (de 1/04 au 20/05 et du 21/05 au $7 / 07 / 1988$ ) atteignaient 9,1 et $12,6^{\circ} \mathrm{C}$, sans déficit hydrique (pluviométrie totale de $300 \mathrm{~mm}$ durant la période). Durant cette période de mesures, 1 an après le semis, les rangs des 2 espèces étaient encore bien séparés dans l'association. L'évolution, au cours de la repousse, de la structure de ces peuplements associés a été analysée précédemment (Soussana et al, 1995a).

\section{Mesures}

Des prélèvements hebdomadaires ont été réalisés dans chaque micro-parcelle à l'aide d'un emportepièce de $(0,34 \times 0,15) \mathrm{m}$, à une profondeur de $10 \mathrm{~cm}$ environ. À chaque prélèvement, les talles du ray-grass ont été coupées au niveau du sol à l'aide de scalpels ; les stolons et les feuilles de trèfle ont été prélevés après l'élimination des racines. La biomasse des stolons, des folioles et des pétioles chez le trèfle, des limbes et des gaines chez le ray-grass a ensuite été déterminée, pour chaque échantillon, par la pesée d'un sous-échantillon préalablement séché à l'étuve $\left(48 \mathrm{~h}\right.$ à $80^{\circ} \mathrm{C}$ ). Après broyage, la teneur en azote total de la matière sèche a été dosée selon Kjeldhal, sur environ $0,25 \mathrm{~g}$ de poudre végétale.

L'effet bloc étant significatif pour les biomasses et les teneurs en azote, une analyse de variance a été effectuée pour chaque coupe et pour chaque traitement azoté, en considérant comme facteurs le type de culture (pure ou en association), la date de prélèvement et le bloc; la plus petite différence significative correspondante a été indiquée dans les figures 1, 2, 5 et 6 .

\section{Méthode d'estimation du niveau de nutrition azotée chez le ray-grass}

Dans le cas du ray-grass anglais, afin de situer nos résultats par rapport à la loi de dilution de l'azote dans la matière sèche formulée par Salette et Lemaire (1981), nous avons pris comme référence la courbe de Lemaire et Salette (1984) correspondant au niveau de nutrition azotée non limitant pour la croissance chez la fétuque élevée. Cette équation s'applique également à d'autres graminées pérennes, ainsi qu'aux prairies permanentes (Lemaire et al, 1989) :

$$
N=4,8 \text { (MS) }^{-0,32}
$$

avec $N$ : teneur en azote total (\%) et MS = biomasse foliaire (t.ha-1).

Dans le cas d'une culture associée, nous ne connaissons pas la surface au sol occupée par un rang de ray-grass. Nous ne pouvons donc calculer la biomasse par mètre carré, ou par hectare, du raygrass associé. L'équation [1] n'est ainsi pas directement utilisable. Toutefois, si l'on admet que les phénomènes de dilution de l'azote dépendent, en partie, du degré de fermeture du couvert végétal (Lemaire et al, 1991) - et donc de la biomasse totale - , nous pouvons substituter dans l'équation [1] la biomasse totale de l'association en t.ha ${ }^{-1}$ à celle de la graminée :

$$
N=4,8\left(M^{\prime}\right)^{-0,32}
$$

avec $N=$ teneur en azote total du ray-grass (\%) et MS' = biomasse foliaire du ray-grass et du trèfle associés $\left(\right.$ t.ha $\left.{ }^{-1}\right)$.

Un autre mode d'expression de la dilution de l'azote, utilisé par Cruz et Lemaire (1986b) consiste à esti- 
mer le degré de croissance d'un rang, qu'il soit pur ou associé, par sa biomasse ramenée au mètre linéaire. Pour les cultures pures, une tonne de matière sèche par hectare équivalait dans notre essai à $17 \mathrm{~g} \mathrm{MS.m}{ }^{-1}$ (les rangs du ray-grass pur occupant $5,9 \mathrm{~m}$ linéaires par $\mathrm{m}^{2}$ ) ; la relation [1] pouvait donc s'écrire : $N=4,8$ $\left(\mathrm{MS}^{\prime \prime} / 17\right)^{-0,32}$

$$
N=12\left(\mathrm{MS}^{\prime \prime}\right)^{-0,32}
$$

où $\mathrm{MS}$ " représente la biomasse par mètre linéaire du ray-grass $\left(\mathrm{gMS}^{-1} \mathrm{~m}^{-1}\right)$, soit $\mathrm{MS}=(\mathrm{MS} / 17)$.

Ces 2 modes d'expression d'une teneur en azote non limitante pour la croissance du ray-grass, [1] ou [2] et [3], ont été comparés, afin de déterminer l'effet de l'association sur le niveau de nutrition azotée de la graminée. Ce niveau ( $\mathrm{Nn}$, exprimé en pourcentage) a été calculé d'après Lemaire et al (1989), en divisant la teneur en azote mesurée par la teneur en azote non limitante pour la croissance, tirée des équations [1] ou [2] et [3].

\section{Séparation des effets de la compétition racinaire et épigée}

Afin de séparer les effets de la compétition interspécifique racinaire de ceux de la compétition interspécifique épigée, un dispositif analogue à celui utilisé par Martin et Field (1984) a été installé dans l'une des micro-parcelles du dispositif, correspondant au traitement association $\mathrm{N}_{00}$. Trois mois après le semis, à une période où les racines des 2 espèces étaient encore peu développées, des séparations métalliques (en acier galvanisé, d'une longueur de $1 \mathrm{~m}$ ) ont été enfoncées $(r)$ ou non $(R)$, à une profondeur de $20 \mathrm{~cm}$ entre les rangs de trèfle et de ray-grass. Par ailleurs, des plaques métalliques verticales d'une hauteur de $40 \mathrm{~cm}$ ont été diposées soit entre les rangs de trèfle et de ray-grass $(f)$ soit perpendiculairement $(F)$. L'ombrage additionnel créé par ces plaques était comparable dans les 2 traitements. Toutefois, les séparations verticales étaient orientées $(E-W)$ en (f), alors qu'elles étaient orientées $(\mathrm{N}-\mathrm{S})$ en $(\mathrm{F})$.

Quatre traitements ont ainsi été réalisés :

- fr : pas de compétition interspécifique, fR : compétition racinaire seule ;

- Fr : compétition épigée seule, FR : compétition racinaire et épigée.

En octobre, mai et juillet pour chaque rang ( 3 rangs de $1 \mathrm{~m}$ par espèce), la biomasse récoltable (MS) et la teneur en azote total $(\mathrm{N})$ ont été déterminées, comme précédemment, et le niveau de nutrition azotée du raygrass a été calculé selon l'équation [2].

Enfin, des dosages complémentaires des teneurs foliaires en phosphore $(P)$ et en potassium $(K)$ ont été effectués et les indices de nutrition phosphatée $\left(I_{P}\right)$ et potassique $\left(I_{K}\right)$ ont été calculés, d'après Duru (1992), comme :

$$
I_{P}=100(P / P o p t)
$$

où Popt, représente le niveau optimum de $P$, tel que $P o p t=0,24$ N0,64;

$$
I_{K}=100(K / K o p t)
$$

où Kopt, représente le niveau optimum de $K$, tel que $K o p t=1,62 \mathrm{~N}^{0,48}$.

Les résultats correspondant ont été analysés par ANOVA, en considérant les rangs comme des répétitions et en prenant comme facteurs la compétition épigée, la compétition racinaire et la date de prélèvement.

\section{RÉSULTATS}

\section{Analyse de la dilution de l'azote foliaire chez le ray-grass}

\section{En fonction de la biomasse foliaire d'un rang de ray-grass}

La faible croissance du ray-grass témoin $\left(\mathrm{N}_{0}\right)$, ainsi que la diminution rapide de sa teneur en azote, attestent que la fourniture d'azote par le sol était faible durant la première repousse (fig 1A). L'apport de 100 kg. $\mathrm{N} \mathrm{ha}^{-1}$ (N1) a permis de rétablir, durant le début de la première repousse, une nutrition azotée non limitante pour la croissance du ray-grass pur (fig 1B).

Durant la seconde repousse, la fourniture d'azote par le sol n'a pas permis de dépasser $2 \%$ d'azote total chez le ray-grass pur non fertilisé $\left(\mathrm{N}_{00}\right.$, fig $\left.1 \mathrm{C}\right)$. À cette période, un apport de 100 $\mathrm{kg} \mathrm{N} \mathrm{ha}^{-1}$ en début de repousse (traitement $\mathrm{N}_{01}$ ) n'a pas évité une limitation de la croissance par le niveau de nutrition azotée (fig 1D).

L'arrière-effet de l'azote apporté en sortie d'hiver a accru, en début de seconde repousse, la nutrition azotée du ray-grass pur (traitements $\mathrm{N}_{10}$ et $N_{11}$ comparés, respectivement, à $N_{00}$ et $N_{01}$ ) (fig 1). Celle-ci demeure toutefois limitante en fin de seconde repousse de printemps. Dans tous les traitements, l'association avec le trèfle a nettement accru la biomasse du ray-grass ramenée au mètre linéaire (fig 1). Ce gain de croissance semble résulter, au moins en partie, d'une amélioration du niveau de nutrition azotée de la graminée, puisque, comparée à même biomasse d'un rang, la teneur en azote du ray-grass associé est généralement supérieure à celle du raygrass pur (fig 1).

Les ray-grass associés qui ont reçu une fertilisation azotée en début de repousse semblent avoir nettement dépassé le niveau minimum de nutrition azotée qui soit non limitant pour la croissance (fig 1B, D et F). En revanche, sans apport d'azote en début de repousse, la croissance du ray-grass associé a été limitée par son niveau de nutrition azotée (fig $1 \mathrm{~A}, \mathrm{C}$ et $\mathrm{E}$ ). 


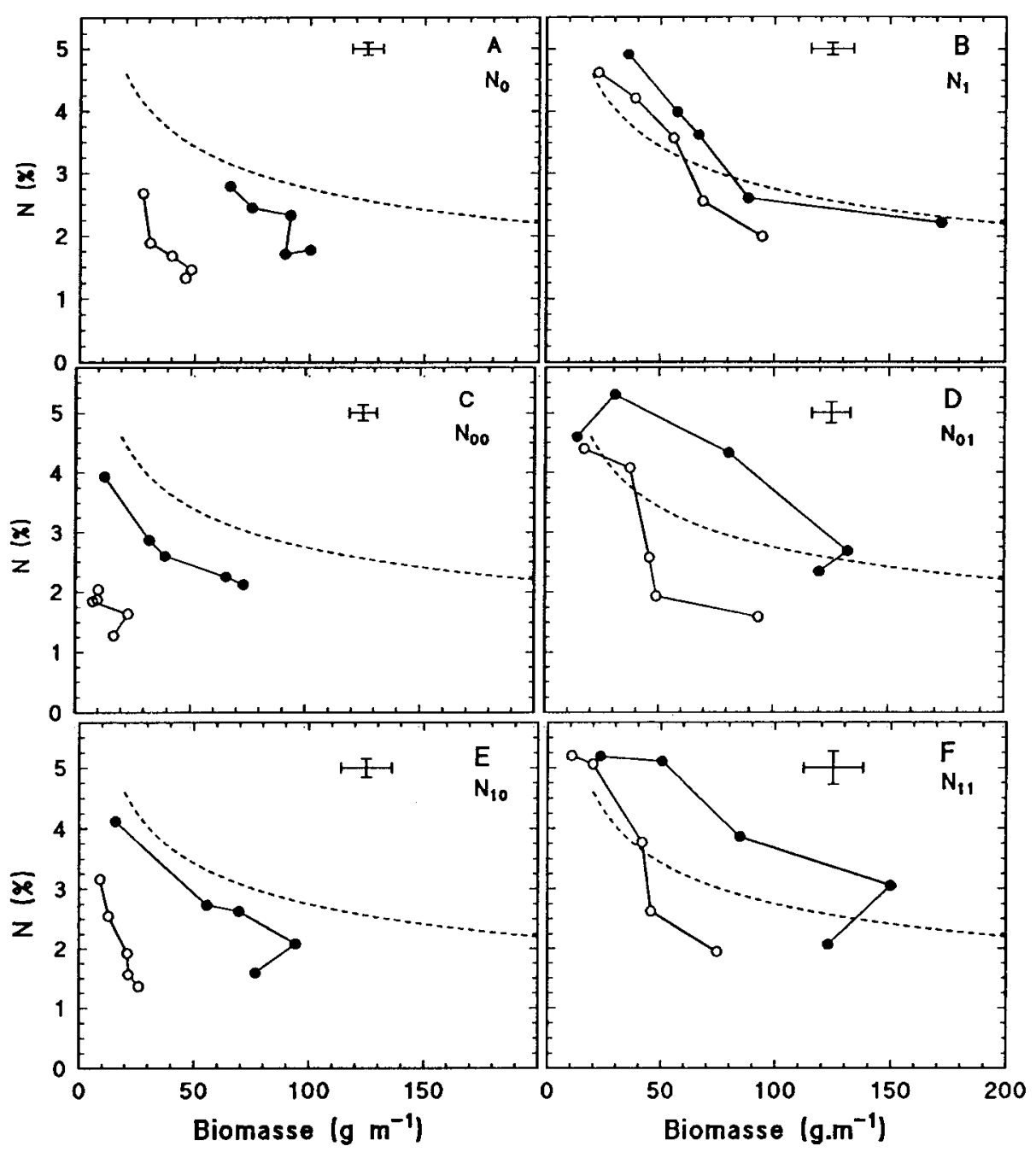

Fig 1. Courbes de dilution de l'azote total $(N)$ dans la matière sèche foliaire au cours de la croissance du ray-grass anglais pur $(O)$ ou associé $(\bullet)$, estimée par sa biomasse foliaire par mètre linéaire $(\mathbf{M S})$ ). (A,B) première repousse de printemps ; $(\mathbf{C}, \mathbf{D}, \mathbf{E}, \mathbf{F})$ seconde repousse de printemps. Pas d'apport d'azote minéral $\left(\mathrm{N}_{0}, \mathrm{~N}_{00}\right) ; 100 \mathrm{~kg} \mathrm{~N} \cdot \mathrm{ha}^{-1}$ au début de la première repousse de printemps $\left(\mathrm{N}_{1}\right.$, $\left.N_{10}\right) ; 100 \mathrm{~kg} \mathrm{~N}$ ha ${ }^{-1}$ au début de la seconde repousse de printemps $\left(N_{0}, N_{01}\right) ; 100 \mathrm{~kg} \mathrm{~N}_{\text {.ha }}{ }^{-1}$ au début des 2 repousses $\left(N_{1}, N_{11}\right)$. La courbe en pointillés correspond à la loi de dilution de l'azote non limitante pour la croissance : $N=12$ (MS")-0,32, dérivée de celle utilisée par Lemaire et al (1989) (cf texte, équation 3). Les barres verticales et horizontales figurent la plus petite différence significative au seuil $P<0,05$.

Le niveau moyen, au cours de la repousse, de nutrition azotée du ray-grass a été calculé d'après l'équation [3] (tableau I). Dans le cas du ray-grass pur non fertilisé, ce niveau a diminué significativement $(P<0,01)$ de la première à la seconde repousse de printemps, passant de 47 à $33 \%$. La fertilisation azotée apportée en début de repousse $\left(100 \mathrm{~kg} \mathrm{~N} \mathrm{ha}^{-1}\right)$ à la culture pure a permis d'atteindre une nutrition azotée optimale $(98 \%)$ durant la première repousse, mais non durant la seconde $(76 \%)$ (tableau I). L'arrièreeffet de l'azote apporté en début de première repousse a permis d'accroître, significativement, le niveau de nutrition azotée du ray-grass pur de $76\left(\mathrm{~N}_{01}\right)$ à $90\left(\mathrm{~N}_{11}\right) \%$, ou (à la limite de la signification) de $33\left(N_{00}\right)$ à $43\left(N_{10}\right) \%$ (tableau I).

Lors des 2 repousses, et pour chaque traitement, l'association avec le trèfle s'est toujours traduite par une augmentation hautement significative du niveau moyen de nutrition azotée du raygrass, calculé d'après l'équation [3] (tableau I). Toutefois, cette augmentation a été plus large (de 41 à $72 \%$, en moyenne) dans les traitements qui n'avaient pas reçu de fertilisation azotée en début de repousse $\left(N_{0}, N_{00}, N_{10}\right)$. En cas d'apport d'azote en début de repousse $\left(N_{1}, N_{01}, N_{11}\right)$, le niveau de nutrition azotée du ray-grass augmente plus faiblement (de 88 à $115 \%$ en moyenne) sous l'effet de la culture associée (tableau I). 
Tableau I. Moyenne et erreur standard du niveau de nutrition azotée moyen (\%) du ray-grass pur ou associé au trèfle, calculés en fonction de la biomasse foliaire de ray-grass par mètre linéaire $\left(\mathrm{m}^{-1}\right)$ ou de la biomasse foliaire totale de l'association $\left(\mathrm{m}^{-2}\right)$.

$\begin{array}{ccc}\text { Coupe } & \text { Traitement } N & \text { Pure } \\ & & \\ 1 & & \\ & N_{0} & 97 \pm 2 \\ 2 & N_{1} & \\ & & 33 \pm 5 \\ & N_{00} & 76 \pm 4 \\ & N_{01} & 43 \pm 5 \\ & N_{10} & 90 \pm 4\end{array}$

$$
\begin{gathered}
\begin{array}{c}
\text { Associée } \\
\left(m^{-1}\right)
\end{array} \\
\\
74 \pm 2^{\star \star} \\
111 \pm 2^{\star *} \\
68 \pm 4^{\star \star} \\
113 \pm 4^{\star *} \\
74 \pm 4^{\star \star} \\
120 \pm 4^{\star *}
\end{gathered}
$$$$
\text { Associée }
$$$$
\left(m^{-2}\right)
$$$$
68 \pm 2^{\star \star \star}
$$$$
95 \pm 2
$$

$66 \pm 4^{\star *}$

$100 \pm 4^{\star \star}$

$64 \pm 4^{\star \star}$

$102 \pm 3^{*}$

Pas d'apport d'azote minéral $\left(N_{0}, N_{00}\right), 100 \mathrm{~kg} \mathrm{~N} . h a^{-1}$ au début de la première repousse de printemps $\left(N_{1}, N_{10}\right), 100 \mathrm{~kg} \mathrm{~N}$.ha-1 au début de la seconde repousse de printemps $\left(N_{0}, N_{01}\right), 100 \mathrm{~kg} \mathrm{~N}$.ha-1 au début des 2 repousses de printemps $\left(N_{1}, N_{11}\right)$. Les notations à un indice $\left(N_{0}, N_{1}\right)$ correspondent à la première repousse ; les notations à 2 indices à la seconde $\left(N_{00}, N_{01}, N_{10}, N_{11}\right) .{ }^{* *},{ }^{*}$ : effet hautement significatif $(P<0,01)$, ou significatif $(P<0,05)$, de la culture en association sur le niveau moyen de nutrition azotée du raygrass (ANOVA pour chaque coupe et pour chaque traitement azoté. Facteurs : culture pure ou associée, date d'observation au cours de la repousse, bloc).

\section{En fonction de la biomasse foliaire totale}

L'expression des teneurs en azote en fonction de la biomasse foliaire totale par hectare est strictement équivalente pour les cultures pures, puisqu'elle correspond à un simple changement d'unités. En revanche, la prise en compte de la biomasse foliaire totale (trèfle plus ray-grass) de l'association (équation [2]) modifie l'évaluation du niveau de nutrition azotée du ray-grass associé. Ainsi, comparées à même biomasse foliaire totale de l'association, les teneurs en azote du raygrass associé ne dépassent guère le niveau optimum de nutrition, considéré comme non limitant pour la croissance (fig 2).

Comparés à même biomasse foliaire totale, les écarts de teneur en azote entre ray-grass pur et associé sont généralement moindres. Les différences restent souvent significatives durant la seconde repousse (fig $2 \mathrm{C}, \mathrm{D}, \mathrm{E}$ et $\mathrm{F}$ ), ainsi que durant la première repousse dans le témoin non fertilisé (fig 2A). En revanche, en cas d'apport d'azote en sortie d'hiver, les courbes de dilution de l'azote des ray-grass purs et associés sont voisines et toutes deux proches de la courbe optimale, non limitante pour la croissance (fig 2B).

Le niveau moyen de nutrition azotée du raygrass associé a été calculé cette fois d'après l'équation [2]. En comparaison de la culture pure, la culture associée a augmenté, de façon significative, le niveau de nutrition azotée des traitements non fertilisés en début de repousse (de 42 à $66 \%$, tableau I). L'amélioration du niveau de nutrition azotée de la graminée par l'association a été plus faible (de 88 à $99 \%$, en moyenne) dans les traitements fertilisés en début de repousse et n'est, en particulier, pas significative pour le traitement $\mathrm{N}_{1}$ (tableau I). Calculé en fonction de la biomasse foliaire totale, le niveau moyen de nutrition azotée du ray-grass ne dépasse pas $100 \%$ (tableau I).

\section{Teneur en azote des feuilles et des stolons de trèfle}

\section{Rapport pondéral (limbes/pétioles) et teneur en azote des feuilles de trèfle}

La teneur en azote total des limbes (5 à 6\%) étant nettement supérieure à celle des pétioles (3 à 4\%) (Arregui, 1990), la teneur en azote de la biomasse foliaire du trèfle dépend linéairement du rapport pondéral (limbes/pétioles) (fig 3). Ce dernier paramètre, qui explique plus de $70 \%$ de la variabilité totale de la teneur en azote des feuilles du trèfle pur ou associé (fig 3 ), diminue au cours de la repousse, en fonction de l'accumulation de biomasse foliaire (fig 4). En effet, si I'on excepte la dernière date d'observation avant coupe, pour laquelle la sénescence des feuilles de trèfle n'était pas négligeable (Soussana et al, 1995a), le rapport pondéral (limbes/pétioles) du trèfle pur ou associé diminue selon une loi puis- 


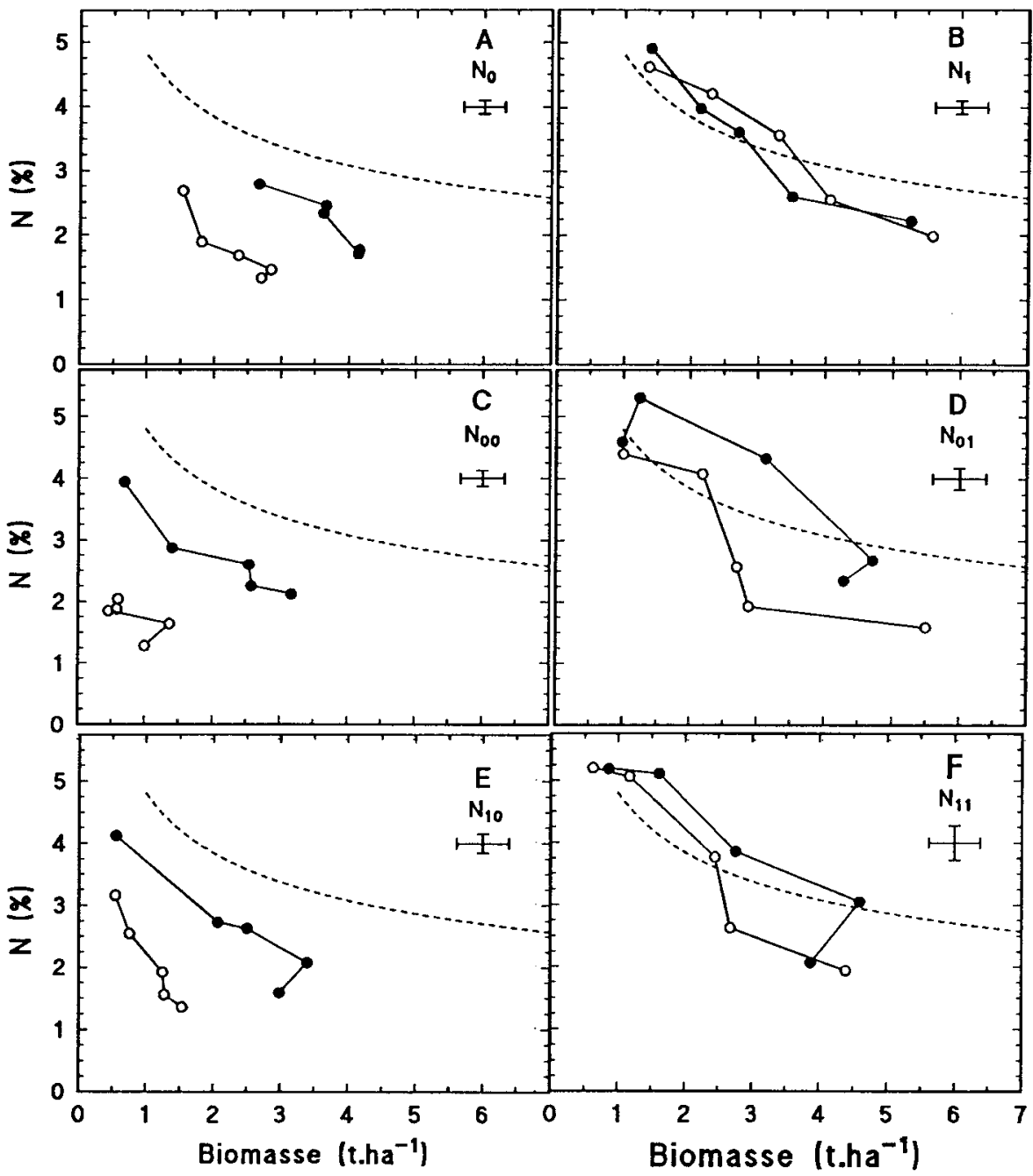

Fig 2. Courbes de dilution de l'azote total dans la matière sèche foliaire au cours de la croissance du ray-grass anglais pur $(O)$ ou associé (๑), estimée par la biomasse foliaire totale par hectare du peuplement pur ou associé. La courbe en pointillés correspond à la loi de dilution de l'azote non limitante pour la croissance : $N=4,8(\mathrm{MS})^{-0,32}$, dérivée de celle proposée par Lemaire et al (1989) (cf texte, équation 1). Les barres verticales et horizontales figurent la plus petite différence significative au seuil $P<0,05$. Mêmes symboles que dans la figure 1.

sance en fonction de la biomasse foliaire totale du couvert végétal (fig 4).

Afin d'isoler les effets concernant la nutrition azotée du trèfle de l'évolution du rapport

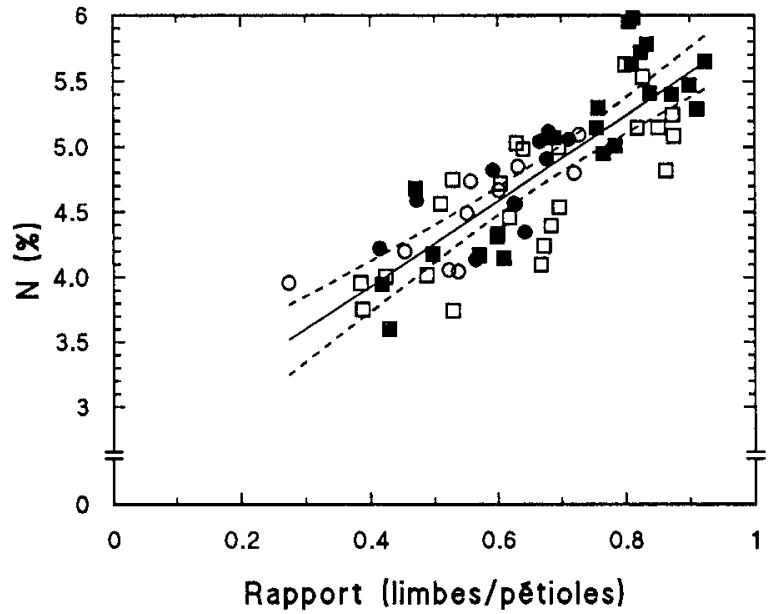

(limbes/pétioles), l'analyse de la dilution de l'azote a été poursuivie sur les limbes de la légumineuse.

\section{Dilution de l'azote des limbes en fonction de la biomasse foliaire d'un rang de trèfle}

Dans le cas du trèfle pur, l'apport d'une fertilisation azotée n'a pas modifié significativement la

Fig 3. Teneur en azote total des feuilles de trèfle blanc en fonction du rapport pondéral (limbes/pétioles) (LP) dans la biomasse foliaire du trèfle pur (symboles ouverts) ou associé (symboles fermés). $(\mathrm{O}, \mathbf{\Theta})$, première repousse de printemps ; $(\square, \square)$, seconde repousse de printemps. La régression linéaire figurée $(-)$ et son erreur standard à $(P<0,05)(---)$ correspondent à l'équation :

$N=(3,0 \pm 0,2)(\mathrm{LP})+(2,7 \pm 0,2) ; n=60 ; r=0,847 ; P<0,001$. 


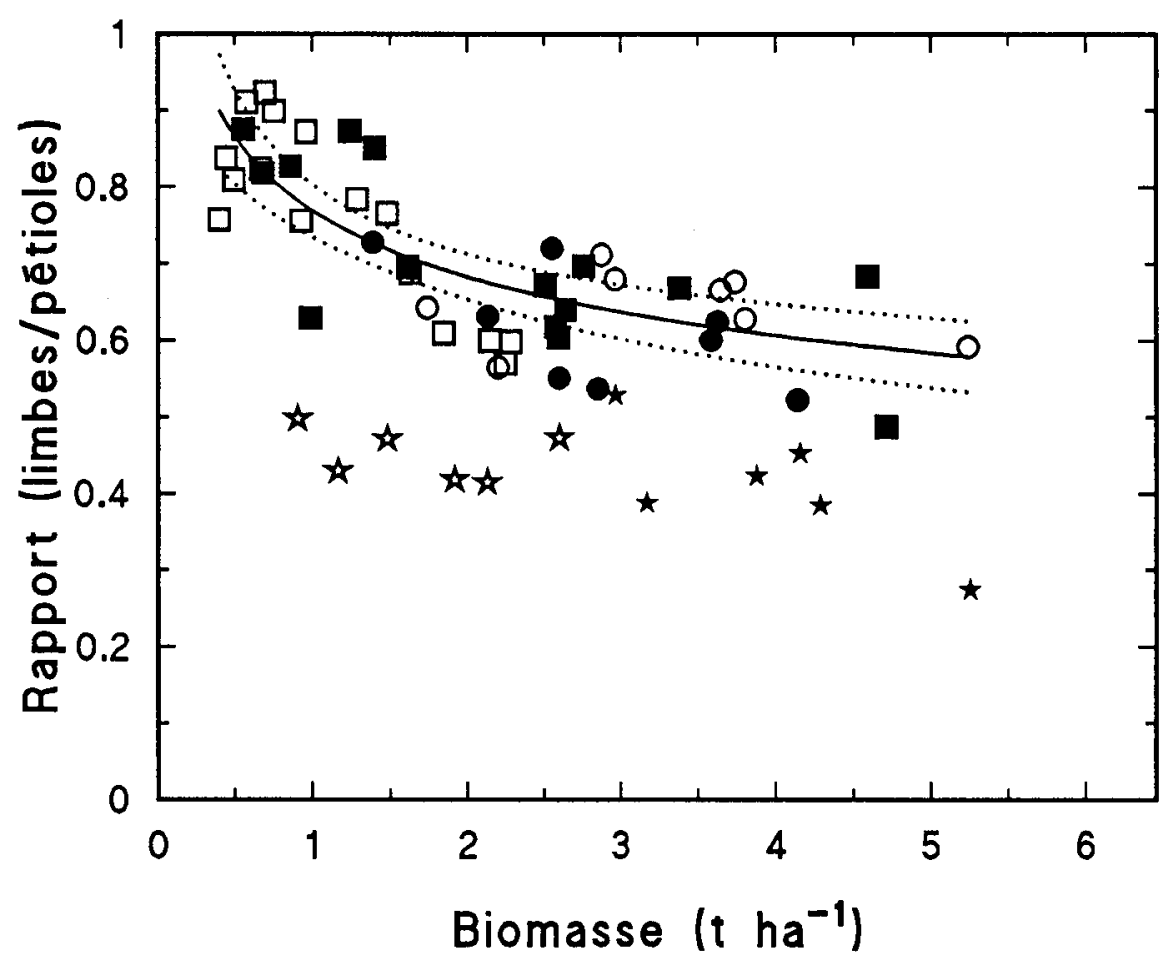

Fig 4. Évolution du rapport pondéral (limbes/pétioles) en fonction de la biomasse foliaire du trètle blanc pur $(O, \square)$ ou de l'association $(\boldsymbol{\bullet}, \mathbf{\square})$, lors de la première $(O, \bullet)$ ou de la seconde $(\square, \boldsymbol{\square})$ repousse de printemps. Les données de rapport pondéral (limbes/pétioles) (LP) des traitements purs et associés ont été ajustées, à une loi puissance (moyenne \pm erreur standard) :

$$
L P=(0,77 \pm 0,01) \mathrm{MS}-(0,17 \pm 0,02) ; n=48 ; r=0,753 ; P<0,001)
$$

Les points qui correspondent au dernier prélèvement avant coupe $(\star, \star)$ ne sont pas présentés du fait d'une sénescence foliaire à ces dates (cf texte).

biomasse foliaire (fig 5). Inversement, en culture associée, les apports d'azote en début de repousse $\left(\mathrm{N}_{1}, \mathrm{~N}_{01}, \mathrm{~N}_{11}\right)$ ont conduit à une réduction de la biomasse foliaire du trèfle (fig 5 ), décrite précédemment (Soussana et al, 1995a).

Les teneurs en azote des trèfles purs et associés ont été similaires lors de la première repousse, malgré une biomasse foliaire plus importante dans la culture pure (fig 5A et B). Durant la seconde repousse, la culture en association a réduit la teneur en azote des limbes de trèfle dans les traitements $N_{01}, N_{10}$ et $N_{11}$ (fig $5 D, 5 E$ et $5 F$ ). Dans ces traitements fertilisés, les limbes du trèfle associé étaient donc plus pauvres en azote, à même biomasse foliaire de la légumineuse, que ceux du trèfle pur.

\section{Dilution de l'azote des limbes en fonction de la biomasse foliaire totale}

La comparaison, à même biomasse foliaire totale (celle du ray-grass plus celle du trèfle, dans le cas de l'association), n'indique aucun effet systématique de la culture en association sur la teneur en azote des limbes de trèfle (fig 6). Ce mode d'expression des résultats permet de montrer la faible décroissance de la teneur en azote total (N) des limbes du trèfle pur ou associé, en fonction de la biomasse foliaire totale du peuplement (MS, en t.ha ${ }^{-1}$ ) (fig 6). Une loi puissance résume, pour l'ensemble des données expérimentales, cette décroissance avec, toutefois, une assez forte variabilité résiduelle :

$$
\begin{gathered}
\mathrm{N}=(5,6 \pm 0,05) \mathrm{MS}^{-}(0,036 \pm 0,010) \\
(n=60 ; r=0,436 ; P<0,001)
\end{gathered}
$$

\section{Teneur en azote des stolons de trèfle}

La teneur moyenne en azote des stolons a été plus faible lors de la seconde repousse de printemps que lors de la première (tableau II). La date de prélèvement a eu un effet significatif dans le cas de 2 traitements $\left(\mathrm{N}_{1}\right.$ et $\mathrm{N}_{11}$, tableau II), mais la teneur en azote des stolons n'a pas diminué de manière régulière au cours de la repousse. $\mathrm{La}$ culture en association a réduit la teneur en azote des stolons dans les traitements qui ont reçu une fertilisation azotée, que celle-ci ait été pratiquée en début de repousse $\left(\mathrm{N}_{1}, \mathrm{~N}_{01}\right)$, ou à la coupe précédente $\left(\mathrm{N}_{10}\right)$ (tableau II). 


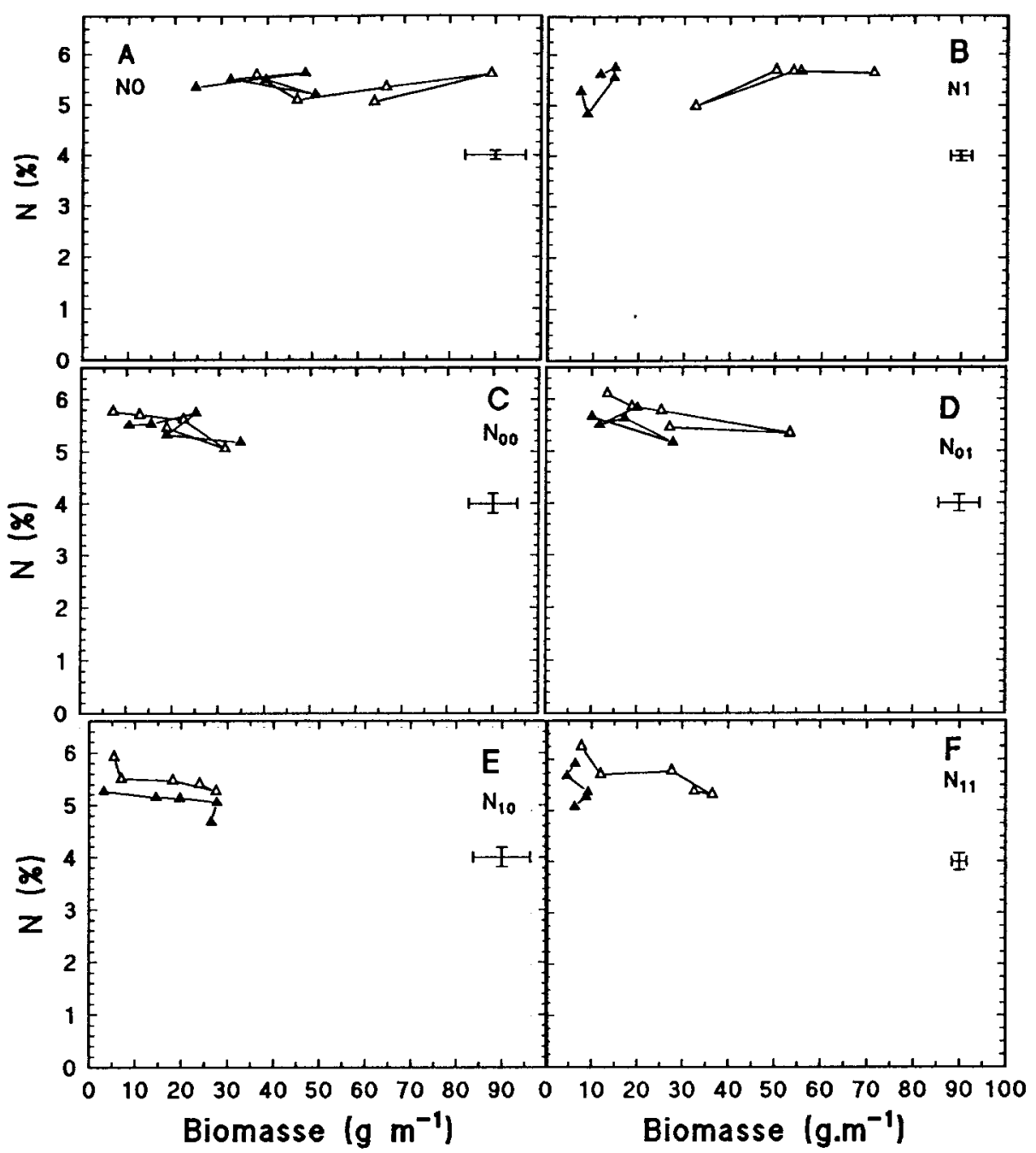

Fig 5. Courbes de dilution de l'azote total des limbes au cours de la croissance du trèfle blanc pur $(\Delta)$ ou associé ( $\mathbf{\Delta}$ ), estimée par sa biomasse foliaire par mètre linéaire. Les barres verticales et horizontales figurent la plus petite différence significative au seuil $P<$ 0,05 . Mêmes symboles que dans la figure 1.

\section{Effets de la compétition interspécifique racinaire et épigée}

Comme ils ne présentent pas d'interactions significatives, les effets de la compétition interspécifique épigée et racinaire ont été analysés séparément.

\section{Compétition épigée}

Dans le traitement étudié $\left(\mathrm{N}_{00}\right)$, la compétition pour la lumière exercée par le trèfle sur le raygrass a réduit la biomasse foliaire de la graminée (ANOVA, $P<0,05$ ), sans changement significatif de son niveau de nutrition azotée, ni de ses indices de nutrition $P$ et $K$ (tableau III). La compétition pour la lumière exercée par le ray-grass n'a eu, en revanche, aucun effet significatif sur le trèfle (tableau III).

\section{Compétition racinaire}

La compétition interspécifique racinaire a conduit à une diminution significative des indices de nutrition pour le phosphore $\left(I_{P}\right)$ et le potassium $\left(I_{K}\right)$ chez le trèfle et à leur augmentation (significative seulement pour $I_{P}$ ) chez le ray-grass (tableau III).

La compétition racinaire exercée par le raygrass a réduit la biomasse récoltable du trèfle, sans modification de sa teneur en azote total (tableau III). Les interactions avec la date d'observation sont, dans ce cas, significatives et font l'objet du tableau IV. En juillet, à la dernière date d'observation, la compétition (ou, dans ce cas, la synergie) racinaire avec le trèfle a augmenté significativement la biomasse, les exportations d'azote et le niveau de nutrition azotée du raygrass (tableau IV). 


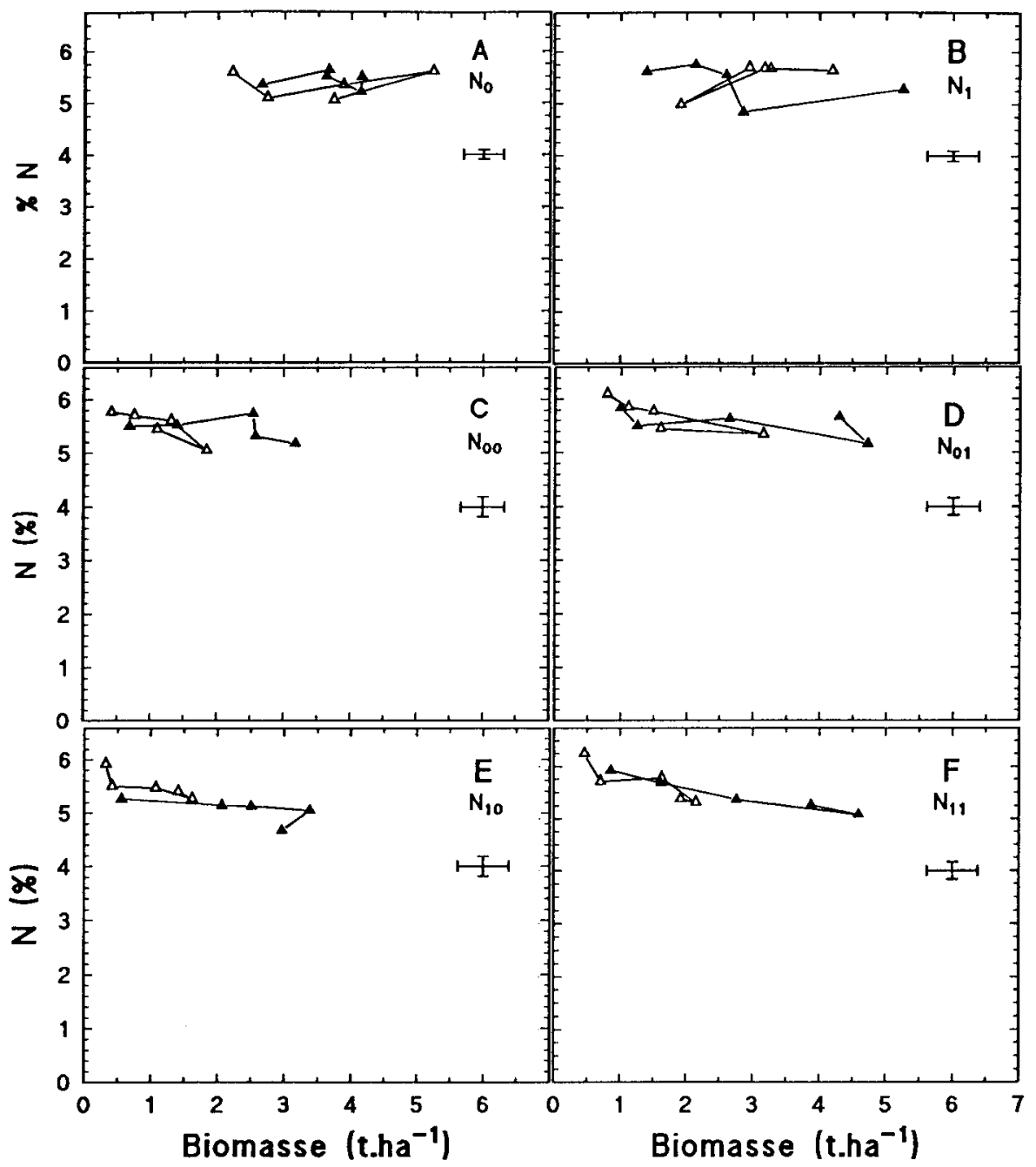

Fig 6. Courbes de dilution de l'azote total des limbes au cours de la croissance du trèfle blanc pur $(\Delta)$ ou associé $(\mathbf{\Delta})$, estimée par la biomasse foliaire totale par hectare du peuplement. Les barres verticales et horizontales figurent la plus petite différence significative au seuil $P<0,05$. Mêmes symboles que dans la figure 1 .

Tableau II. Teneur en azote total des stolons du trèfle blanc pur ou associé.

\begin{tabular}{|c|c|c|c|c|c|}
\hline \multirow[t]{2}{*}{ Repousse } & \multirow{2}{*}{$\begin{array}{c}\text { Fertilisation } \\
\text { azotée }\end{array}$} & \multicolumn{2}{|c|}{ Teneur en azote total (\%) } & \multirow[t]{2}{*}{ Association } & \multirow{2}{*}{$\begin{array}{l}\text { Degré de } \\
\text { repousse }\end{array}$} \\
\hline & & Pure & Associée & & \\
\hline \multirow[t]{2}{*}{1} & $\mathrm{~N}_{0}$ & $3,12 \pm 0,08$ & $3,02 \pm 0,09$ & NS & NS \\
\hline & $\mathrm{N}_{1}$ & $3,31 \pm 0,08$ & $2,98 \pm 0,08$ & $\star \star$ & $\star \star$ \\
\hline \multirow[t]{4}{*}{2} & $\mathrm{~N}_{0}$ & $2,75 \pm 0,05$ & $2,71 \pm 0,06$ & NS & NS \\
\hline & $N_{1}$ & $2,97 \pm 0,05$ & $2,52 \pm 0,07$ & $\star \star$ & NS \\
\hline & $\mathrm{N}_{10}$ & $2,76 \pm 0,05$ & $2,55 \pm 0,07$ & * & NS \\
\hline & $\mathrm{N}_{11}$ & $2,97 \pm 0,04$ & $2,61 \pm 0,07$ & $\star \star$ & * \\
\hline
\end{tabular}

${ }^{* *},{ }^{*}$ : effet hautement significatif $(P<0,01)$ ou significatif $(P<0,05)$ de la culture en association sur la teneur en azote des stolons de trèfle (ANOVA pour chaque repousse et pour chaque traitement azoté. Facteurs : culture pure ou associée, date d'observation au cours de la repousse, bloc). L'effet bloc n'est pas significatif. L'effet fertilisation azotée (ANOVA pour chaque repousse) n'est pas significatif. Mêmes notations que pour le tableau $\mathrm{l}$. 
Tableau III. Effets de la compétition interspécifique sur la biomasse produite (MS), les teneurs en azote (N), phosphore $(P)$ et potassium $(K)$ des parties récoltées, le niveau de nutrition azotée (Nn), calculé d'après l'équation [3] et les indices de nutrition phosphatée $\left(I_{P}\right)$ et potassique $\left(I_{K}\right)$ chez le ray-grass et chez le trèfle du traitement $N_{00}$.

\begin{tabular}{|c|c|c|c|c|c|c|c|}
\hline \multirow[t]{2}{*}{...... } & \multirow[b]{2}{*}{$M S\left(g m^{-1}\right)$} & $\ldots$ & \multirow{2}{*}{$N n(\%)$} & - - & \multirow{2}{*}{$I_{P}(\%)$} & \multicolumn{2}{|l|}{$\cdots$} \\
\hline & & $N(\%)$ & & $P(\%)$ & & $K(\%)$ & $I_{K}(\%)$ \\
\hline & - - & $-\ldots$ & -.. & - & - --. & -.. & -- \\
\hline \multicolumn{8}{|c|}{ Ray-grass } \\
\hline$f$ & $52 \pm 4$ & $2,0 \pm 0,16$ & $56 \pm 2,8$ & $0,308 \pm 0,01$ & $85 \pm 3$ & $3,3 \pm 0,07$ & $150 \pm 6$ \\
\hline$F$ & $48 \pm 4^{*}$ & $2,2 \pm 0,16^{*}$ & $59 \pm 2,7$ & $0,318 \pm 0,01$ & $83 \pm 3$ & $3,3 \pm 0,10$ & $143 \pm 6$ \\
\hline$r$ & $50 \pm 4$ & $2,1 \pm 0,17$ & $58 \pm 3,3$ & $0,302 \pm 0,01$ & $81 \pm 3$ & $3,2 \pm 0,08$ & $144 \pm 5$ \\
\hline $\mathrm{R}$ & $50 \pm 4$ & $2,1 \pm 0,14$ & $57 \pm 2,1$ & $0,324 \pm 0,01^{\star}$ & $87 \pm 3^{*}$ & $3,4 \pm 0,09$ & $150 \pm 7$ \\
\hline \multicolumn{8}{|c|}{ Trèfle } \\
\hline $\mathfrak{f}$ & $27 \pm 3$ & $4,0 \pm 0,12$ & - & $0,365 \pm 0,03$ & $62 \pm 4$ & $3,7 \pm 0,18$ & $118 \pm 6$ \\
\hline$F$ & $30 \pm 4$ & $4,1 \pm 0,12$ & - & $0,360 \pm 0,02$ & $61 \pm 3$ & $3,7 \pm 0,16$ & $117 \pm 6$ \\
\hline$r$ & $31 \pm 4$ & $4,0 \pm 0,13$ & - & $0,377 \pm 0,02$ & $64 \pm 3$ & $3,9 \pm 0,17$ & $125 \pm 6$ \\
\hline $\mathrm{R}$ & $26 \pm 2^{\star}$ & $4,1 \pm 0,11$ & - & $0,348 \pm 0,03^{\star}$ & $58 \pm 4^{\star}$ & $3,5 \pm 0,16^{\star}$ & $110 \pm 5^{*}$ \\
\hline
\end{tabular}

Les résultats correspondent à la moyenne et à l'erreur standard de 3 coupes $(10 / 87,05 / 88,07 / 88) ; f, r$, respectivement : pas de compétition entre parties épigées ou racinaires des 2 espèces; $F, R$, respectivement : compétition entre parties épigées ou racinaires des 2 espèces. *, effet significatif $(P<0,05)$ de la compétition interspécifique épigée $(F)$ ou racinaire (R) (ANOVA, facteurs : compétition épigée, compétition racinaire, date).

Tableau IV. Biomasse récoltée (MS, g. $\left.\mathrm{m}^{-1}\right)$, azote exporté $\left(\mathrm{Ne}, \mathrm{g} \cdot \mathrm{m}^{-1}\right)$ et niveau de nutrition azotée (Nn) de rangs de ray-grass et de trèfle du traitement $N_{00}$, soumis $(R)$ ou non ( $r$ à une compétition interspécifique racinaire lors de 3 récoltes successives, en octobre, mai et juillet, 6,12 et 14 mois après le semis, respectivement.

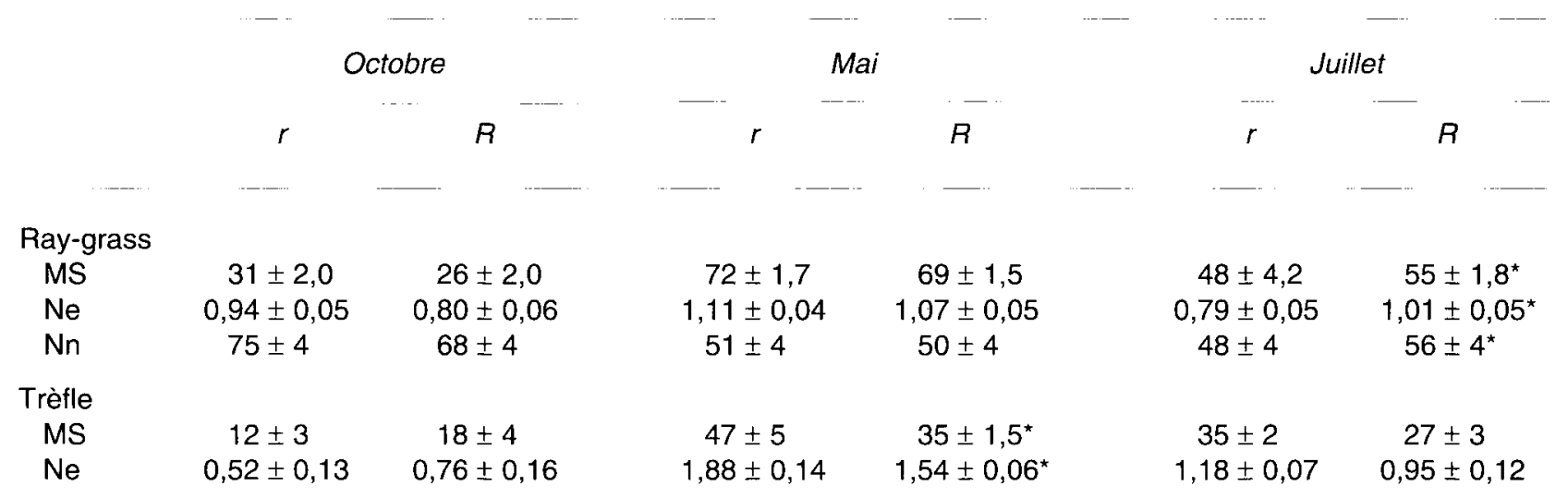

Les résultats correspondent à la moyenne et à l'erreur standard de 3 rangs. * effet significatif $(P<0,05)$ de la compétition épigée (F) ou racinaire $(R)$ (ANOVA, à chaque date, facteur : compétition racinaire).

\section{DISCUSSION}

\section{Diagnostic du niveau de nutrition azotée dans une association prairiale graminée-trèfle}

Le niveau de nutrition azotée des monocultures fourragères peut être caractérisé grâce à l'analyse des courbes de dilution de l'azote au cours de la croissance (Salette et Lemaire, 1981 ; Lemaire et Salette, 1984). L'application de cette méthode au cas d'une association prairiale à base de trèfle suscite un certain nombre de difficultés.

D'une part, les teneurs en azote foliaire du trèfle étant nettement supérieures à celles de la graminée associée, la teneur en azote des fourrages récoltés dépendra notablement de la proportion de trèfle dans l'association. Contrairement au cas de prairies permanentes plurispécifiques (Duru, 1992), l'estimation d'un niveau de nutrition azotée ne pourra donc être basée sur la simple teneur en azote du fourrage récolté. 
Pour des associations présentant une structure en rangs alternés, la comparaison des teneurs en azote peut être effectuée sur la base de la biomasse foliaire par mètre linéaire d'un rang (Cruz et Lemaire, 1986b), ou sur la base de la biomasse foliaire totale de l'association. La comparaison de ces 2 méthodes fait apparaître des différences importantes. Le niveau moyen de nutrition azotée du ray-grass associé est ainsi systématiquement plus élevé lorsque son calcul repose sur la biomasse d'un rang (tableau I).

En particulier, durant la seconde repousse, le niveau moyen de nutrition azotée, basé sur la biomasse foliaire d'un rang de ray-grass associé, dépasse significativement l'optimum dans les traitements fertilisés ( 113 et $120 \%$ en $\mathrm{N}_{01}$ et $\mathrm{N}_{11}$, respectivement, tableau I), ce qui devrait se traduire par une accumulation foliaire de nitrate (Gastal et Saugier, 1986). Pourtant, durant la seconde repousse, l'accumulation foliaire de nitrate était négligeable chez le ray-grass associé (moins de $0,1 \%$ de $\mathrm{N}-\mathrm{NO}_{3}{ }^{-}$dans les limbes, Arregui, 1990). Le niveau de nutrition azotée semble donc être surestimé lorsqu'il est calculé à partir de la biomasse foliaire d'un rang de raygrass associé.

II n'est pas sûr qu'une même loi de dilution s'applique à un rang de ray-grass pur et à un rang de ray-grass associé. Ce dernier colonise, en effet, les rangs adjacents de trèfle de plus faible biomasse foliaire (figs 1 et 6 ) et présente donc un feuillage moins dense et probablement mieux éclairé. Or la teneur en azote foliaire dépend, en partie, de l'éclairement reçu dans le couvert végétal (Charles-Edwards et al, 1987 ; Lemaire et al, 1991 ; Soussana et al, 1995b). Une augmentation de l'éclairement moyen pourrait donc s'accompagner d'une moindre dilution de l'azote foliaire chez le ray-grass associé. La courbe de dilution de référence serait alors intermédiaire (Lemaire, 1995) entre la courbe obtenue en culture pure (donnée dans la figure 1) et celle qui serait obtenue sur des ray-grass isolés, sans compétition pour la lumière.

La prise en compte de la biomasse foliaire totale du peuplement associé est de nature à réduire ces erreurs, notamment lorsque les feuilles du trèfle et de la graminée associée sont étroitement imbriquées, comme c'est généralement le cas dans la pratique agricole. En effet, l'éclairement moyen des feuilles de ray-grass dépend alors de la densité moyenne du feuillage, soit, en première approximation, de la biomasse foliaire totale du couvert associé. Ce paramètre serait donc acceptable pour comparer les teneurs en azote de la graminée associée (fig 2).

Toutefois, l'éclairement moyen des feuilles diffère entre une culture pure et une association de même biomasse foliaire (Sinoquet et al, 1990). Un diagnostic plus précis de la nutrition azotée nécessite, par conséquent, la détermination simultanée, pour chaque espèce associée, des profils verticaux de teneur en azote et d'éclairement des feuilles (Soussana et al, 1995b). Un diagnostic simplifié, reposant sur la teneur en azote des feuilles bien éclairées, situées au sommet du couvert, pourrait également être envisagé.

L'élongation des pétioles de trèfle est régulée par un mécanisme photo-morphogénétique (Solangaarachchi et Harper, 1987 ; VarletGrancher et al, 1989). Au cours d'une repousse, la taille des limbes augmente moins rapidement que celle des pétioles et la longueur de ces derniers s'ajuste à environ $60 \%$ de celle des feuilles du ray-grass (Davies et Evans, 1990). Ceci explique que le rapport pondéral (limbes/pétioles) varie chez le trèfle associé en fonction de la biomasse foliaire totale (trèfle plus ray-grass) de l'association (fig 4)

Cette réduction du rapport pondéral (limbes/pétioles) s'accompagne (fig 3) d'une diminution de la teneur en azote des feuilles de trèfle. De même, chez la luzerne, la baisse au cours de la repousse du rapport (feuilles/tiges) a été observée, avec comme conséquence une dilution de l'azote foliaire (Lemaire et al, 1985).

Ce phénomène de dilution de l'azote a, également, d'autres causes puisque la teneur en azote des limbes de trèfle diminue au cours de la repousse (fig 6). La dilution de l'azote dans les limbes de trèfle serait reliée à la diminution de leur éclairement moyen (Soussana et al, 1995b) au cours de la repousse, du fait de l'auto-ombrage et de l'ombrage exercé par la graminée. Un vieillissement des limbes de la légumineuse pourrait également contribuer à ce phénomène de dilution ; toutefois, chez la luzerne, l'éclairement et non l'âge - explique les variations de la teneur en azote total des limbes (Lemaire et al, 1991).

\section{Effet de l'association sur le niveau de nutrition azotée du ray-grass}

Qu'il soit calculé sur la base du mètre linéaire, ou sur la base du mètre carré, le niveau moyen de nutrition azotée du ray-grass s'est accru, le plus souvent de manière hautement significative, en cas de culture associée avec le trèfle (tableau I). 
Ce résultat confirme donc, globalement, l'effet améliorateur de l'association sur la nutrition azotée de la graminée (Broadbent et al, 1982 ; Arnaud et Niqueux, 1986). Cet effet améliorateur n'a toutefois pas été significatif (tableau I, comparaison à même biomasse foliaire totale de l'association) après fertilisation azotée en première repousse de printemps (traitement $N_{1}$ ). Dans ce cas, le taux de trèfle dans l'association a chuté rapidement dès la sortie de l'hiver, atteignant $7 \%$ en fin de première repousse de printemps (Soussana et al, 1995a). Ce faible taux de trèfle explique sans doute l'impact limité de l'association sur la nutrition azotée de la graminée dans ce traitement.

Le dispositif expérimental en substitution utilisé pour cette étude permet d'analyser les effets du remplacement d'une partie de la compétition intra-spécifique (exercée par le ray-grass) par une compétition inter-spécifique (entre trèfle et raygrass) (de Wit et al, 1966 ; Cousens et O'Neill, 1993 ; Sackville-Hamilton, 1994). L'effet améliorateur de l'association sur le niveau de nutrition azotée de la graminée correspond donc au bilan des effets de compétition intra- et interspécifique.

L'utilisation de séparations entre rangs adjacents de l'association permet, en revanche, d'analyser l'effet propre de la compétition interspécifique épigée ou racinaire. Nous n'avons pas obtenu, en moyenne sur 3 repousses, d'effet significatif de la compétition interspécifique racinaire sur le niveau de nutrition azotée $(N n)$ du ray-grass $\mathrm{N}_{00}$ (tableau III). Toutefois, lors de la dernière repousse étudiée (juillet), la biomasse, la quantité d'azote accumulée et le niveau de nutrition azotée des limbes des ray-grass ont été significativement plus importants lorsque les racines de ray-grass et de trèfle n'étaient pas séparées par une cloison verticale (tableau IV)

Ce sous-essai n'a été mis en place que sur une micro-parcelle. De plus, seules les racines situées dans l'horizon 0-20 cm étaient séparées par des cloisons verticales. Enfin, en minimisant l'imbrication des racines des 2 espèces, la structure en lignes alternées de notre dispositif expérimental a pu réduire l'ampleur des interférences racinaires.

Malgré ces limitations, l'apparition progressive, déjà observée par Bakhuis et Kleter (1965), d'une amélioration de la nutrition azotée du raygrass, liée à la colonisation racinaire des rangs de trèfle adjacents, permet de confirmer que des interférences (Harper, 1977) racinaires entre espèces sont bien responsables de l'effet amélio- rateur de l'association sur la nutrition azotée de la graminée.

Deux hypothèses, non exclusives, permettent de rendre compte de la nature de ces interférences racinaires. D'une part, la substitution de la compétition intraspécifique pour l'azote entre rangs adjacents de ray-grass par une compétition interspécifique plus faible (entre les rangs adjacents de trèfle et de ray-grass) pourrait accroître l'absorption d'azote minéral par la graminée (Vallis et al, 1967). D'autre part, la décomposition des tissus sénescents du trèfle, ou encore l'exudation de composés azotés par ses racines nodulées, libéreraient dans le sol une fraction de l'azote fixé qui deviendrait ainsi accessible à la graminée (Haynes, 1980 ; Harris, 1987).

Nos résultats ne permettent pas de départager ces 2 hypothèses, puisque l'on peut estimer soit que la graminée a bénéficié du moindre pouvoir de compétition pour l'azote minéral du trèfle (Soussana et al, 1989 ; Faurie, 1994) pour accroître son absorption de l'azote dérivé du sol ou de l'engrais, soit qu'elle a absorbé une fraction de l'azote qui avait été fixé par le trèfle. Pour préciser la source de l'azote supplémentaire absorbé par le ray-grass associé, une étude complémentaire, utilisant la technique de traçage isotopique ${ }^{15} \mathrm{~N}$ et des mesures de l'activité de la nitrate réductase, a été entreprise.

\section{Effet de l'association sur la teneur en azote des feuilles et des stolons de trèfle}

La teneur en azote des limbes du trèfle associé, ainsi que le rapport (limbes/pétioles) varient, comme discuté précédemment, non en fonction de la biomasse d'un rang de trèfle, mais en fonction de la biomasse foliaire totale des peuplements purs ou associés. Ce critère a donc été retenu pour discuter des effets de l'association sur la teneur en azote des limbes de trèfle.

Dans l'ensemble des traitements étudiés, l'association avec le ray-grass anglais n'a pas modifié significativement la teneur en azote des limbes du trèfle (fig 6). De plus, dans le cas du traitement $\mathrm{N}_{00}$, la teneur en azote des limbes du trèfle n'a pas été affectée significativement par la compétition pour la lumière, ou par la compétition racinaire, exercées par le ray-grass (tableau III). On peut donc conclure que l'association avec le ray-grass ne donne pas lieu à une variation notable de la teneur en azote des limbes de trèfle.

L'association diminue légèrement la teneur en azote des stolons dans les traitements recevant 


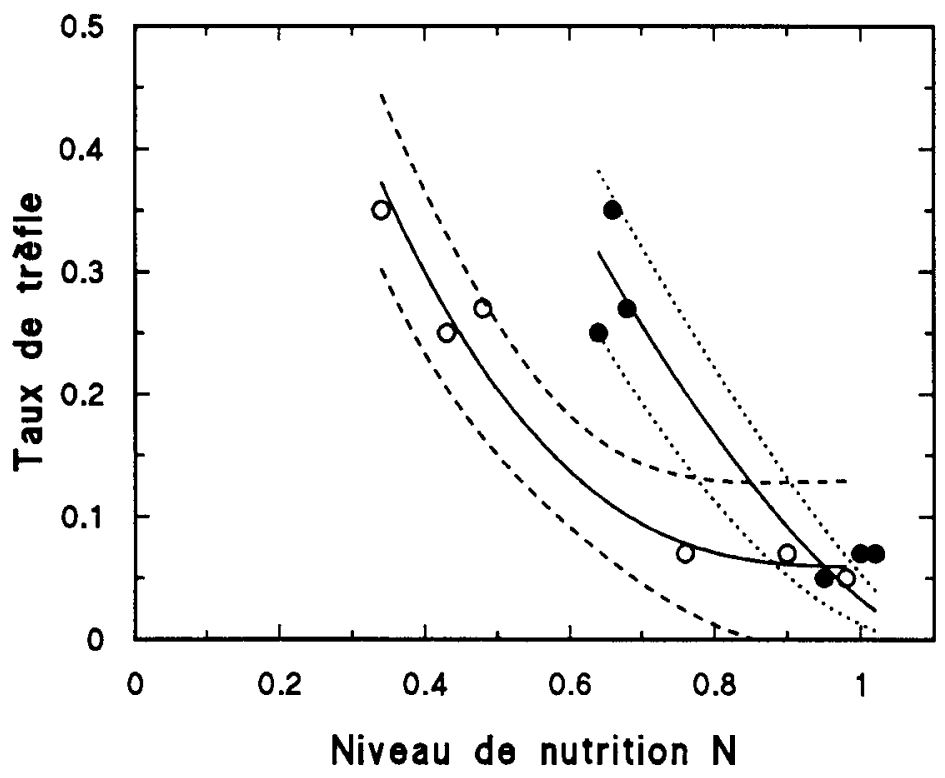

Fig 7. Taux pondéral (TB) de trèfle dans la biomasse foliaire de l'association, en fin de repousse, en fonction du niveau moyen de nutrition azotée $(\mathrm{Nn}) \mathrm{du}$ ray-grass associé (Nna) ou du ray-grass pur recevant la même fertilisation azotée ( $\mathrm{Nnp})$. Les données expérimentales ont été ajustées aux équations suivantes :

$$
\begin{gathered}
T B=(0,060 \pm 0,019)+(1-\mathrm{Nnp})^{(2,79 \pm 0,22)} ; \\
n=6 ; r=0,979 ; P<0,001
\end{gathered}
$$

$T B=(1-\mathrm{Nna})^{(1,48 \pm 0,10)} ; n=6 ; r=0,948 ; P<0,01$

Les lignes en pointillés figurent l'intervalle de confiance de ces régressions au seuil $(P<0,05)$. une fertilisation azotée (tableau II). Cet effet pourrait provenir d'une sur-dilution de l'azote total, du fait d'une accumulation plus forte d'amidon dans les stolons des trèfles associés et fertilisés. En effet, la teneur en amidon, qui peut atteindre $40 \%$ du poids sec, est de 2 à 3 fois plus forte dans les stolons principaux que dans les stolons secondaires (Baur-Höch et al, 1990). Or, du fait d'un phénomène de compétition asymétrique décrit précédemment (Soussana et al, 1995a), l'association a augmenté la proportion de stolons principaux de $35 \%$, en moyenne, dans les traitements recevant une fertilisation azotée (Arregui, 1990).

Par conséquent, des modifications de la morphologie du trèfle (diminution du rapport limbes/pétioles, de la proportion de stolons secondaires) et de l'éclairement de ses limbes rendent compte de l'effet négatif (Broadbent et al, 1982 ; la présente étude) de l'association sur la teneur en azote du trèfle. Un défaut de nutrition azotée de la légumineuse ne peut donc pas expliquer sa fréquente régression (Frame et Newbould, 1986 ; Soussana et al, 1995a) dans les associations recevant une fertilisation azotée.

En revanche, la compétition interspécifique racinaire semble réduire la nutrition phosphopotassique et la biomasse du trèfle et augmenter, inversement, la nutrition $P$ du ray-grass (tableau III). Même en cas d'apport d'une fumure de fond, le moindre pouvoir de compétition du trèfle blanc pour $P$ et pour K (Mouat et Walker, 1959 ; Mouat, 1983) pourrait donc intervenir dans l'équilibre des associations semées.

\section{Conséquences du niveau de nutrition azotée du ray-grass pour l'équilibre de l'association}

La figure 7 montre que le taux de trèfle dans l'association varie en raison inverse $(P<0,01) d u$ niveau de nutrition azotée du ray-grass associé. Les effets d'un apport d'azote minéral sur l'équilibre de l'association sont donc fonction de la modification du niveau de nutrition azotée de la graminée associée qu'ils induisent. Ainsi, dans le cas de notre expérience, un pourcentage appréciable de trèfle (environ $30 \%$ ) n'a été obtenu qu'en l'absence de fertilisation azotée, soit dans des conditions où la graminée associée était nettement carencée en azote (niveau de nutrition azotée de 0,7 environ) (fig 7).

Le taux de trèfle dans l'association varie également en raison inverse $(P<0,001)$ du niveau de nutrition azotée du ray-grass pur recevant la même fertilisation azotée dans les micro-parcelles voisines (fig 7). Par conséquent, si la fertilité azotée de la parcelle est trop importante (niveau de nutrition azotée de la graminée pure supérieure à 0,5 environ), en cas de semis d'une association, le taux de trèfle sera vraisemblablement très faible. Cette relation, établie en un site et pour une saison, ne peut cependant pas être généralisée.

\section{REMERCIEMENTS}

Les auteurs tiennent à remercier $\mathrm{R}$ Delpy pour son aide technique efficace. 


\section{RÉFÉRENCES}

Arnaud R, Niqueux M (1986) Force et faiblesse du trèfle blanc en moyenne montagne. Fourrages 106, 45-66

Arregui MC (1990) Contribution à l'étude des facteurs morphogénétiques et trophiques $(\mathrm{C}, \mathrm{N})$ de la compétition dans les associations trèfle blanc (Trifolium repens $\mathrm{L}$ ) ray-grass anglais (Lolium perenne $\mathrm{L}$ ). Thèse, univ Blaise-Pascal (Clermont II), $171 \mathrm{p}$

Bakhuis JA, Kleter HJ (1965) Some effects of associated growth on grass and clover under field conditions. Neth J Agric Sci 13, 280-310

Baur-Höch B, Mächler F, Nösberger J (1990) Effect of carbohydrate demand on the remobilization of starch in stolons and roots of white clover (Trifolium repens $\mathrm{L}$ ) after defoliation. J Exp Bot 41, 573-578

Birch HF, Dougall HW (1967) Effect of a legume on soil nitrogen mineralization and percentage nitrogen in grasses. Plant Soil 17, 292-296

Böller BC, Nösberger J (1987) Symbiotically fixed nitrogen from field-grown white and red clover mixed with ryegrasses at low levels of $15 \mathrm{~N}$-fertilization. Plant Soil 104, 219-226

Broadbent FE, Nakashima T, Chang GY (1982) Estimation of nitrogen fixation by isotope dilution in field and greenhouse experiments. Agro $J 74$, 625629

Brophy LS, Heichel GH, Russelle MP (1987) Nitrogen transfer from forage legumes to grass in a systematic planting design. Crop Sci 27, 753-758

Charles JP (1982) Utilisation en Suisse du trèfle violet et de la luzerne avec des graminées. Fourrages 90 , 39-56

Charles-Edwards DA, Stutzel H, Ferraris R, Beech DF (1987) An analysis of spatial variation in the nitrogen content of leaves from different horizons within a canopy. Ann Bot 60, 421-426

Cousens R, O'Neill M (1993) Density dependence of replacement series experiments. Oikos 66, 347-352

Cruz P, Lemaire G (1986a) Analyse des relations de compétition dans une association de luzerne (Medicago sativa L) et de dactyle (Dactylis glomerata $L$ ). I. Effets sur les dynamiques de croissance en matière sèche. agronomie 6, 727-734

Cruz P, Lemaire G (1986b) Analyse des relations de compétition dans une association de luzerne (Medicago sativa L) et de dactyle (Dactylis glomerata L). II. Effets sur la nutrition azotée des 2 espèces. agronomie 6, 735-742

Davies A, Evans ME (1990) Effects of spring defoliation and fertilizer nitrogen on the growth of white clover in ryegrass clover swards. Grass Forage Sci 45, 345-356

Duru M (1992) Diagnostic de la nutrition minérale des prairies permanentes au printemps. I. Établissement de références. agronomie 12, 219-233

Faurie O (1994) Interactions carbone-azote dans des associations prairiales graminée (Lolium perenne $L$ )- légumineuse (Trifolium repens $L$ ). Étude d'associations simulées en conditions contrôlées. Thèse doctorat, univ Blaise-Pascal, $203 \mathrm{p}$

Faurie O, Soussana JF (1993) Oxygen-induced recovery from short-term nitrate inhibition of $\mathrm{N}_{2}$ fixation in white clover plants from spaced and dense stands. Physiol Plant 89, 467-475

Frame J, Newbould P (1986) Agronomy of white clover. Adv Agron 40, 1-88

Gastal F, Saugier B (1986) Alimentation azotée et croissance de la fétuque élevée. II. Absorption de l'azote et distribution dans la plante. agronomie 6, 363-370

Gückert A, Damay J, Treillet L, Balandreau J, Bardin $R$, Chalamet A (1983) Étude au champ de la fixation d'azote par le trèfle blanc (Trifolium repens $\mathrm{L}$ ). Fourrages 94, 61-86

Harper JL (1977) The Population Dynamics of Plants. Academic Press, New York

Harris W (1987) Population dynamics and competition. In : White Clover (MJ Baker, WM VWilliams, eds), CAB International, Londres, 203-259

Haynes RJ (1980) Competitive aspects of the grasslegume association. Adv Agron 33, 227-256

Jacquard $P$ (1968) Manifestation et nature des relations sociales chez les végétaux supérieurs. $C E C O$ Plant II, 137-168

Lemaire G, Salette J (1984) Relation entre dynamique de l'azote et dynamique de prélèvement d'azote pour un peuplement de graminées fourragères. I. Étude de l'effet du milieu. agronomie 4, 423-430

Lemaire G, Cruz P, Gosse G, Chartier M (1985) Étude des relations entre la dynamique de prélèvement d'azote et la dynamique de croissance en matière sèche d'un peuplement de luzerne (Medicago sativa L). agronomie 5, 685-692

Lemaire G, Gastal F, Salette J (1989) Analysis of the effect of $N$ nutrition on dry matter yield of a sward by reference to potential yield and optimum $\mathrm{N}$ content. In : Proc XVI Int Grass/ Cong, AFPF, Nice, France, 179-180

Lemaire G, Onillon B, Gosse G, Chartier M, Allirand JM (1991) Nitrogen distribution within a lucerne canopy during regrowth. Relation with light distribution. Ann Bot 68, 483-488

Lemaire G (1995) Ecophysiological approaches to intercropping. In: Ecophysiology of Tropical Intercropping ( $\mathrm{H}$ Sinoquet, P Cruz, eds), INRA, Paris, 9-25

Martin MPLD, Field RJ (1984) The nature of competition between perennial ryegrass and white clover. Grass Forage Sci 39, 247-253

Mariott CA (1988) Seasonal variation in white clover content and nitrogen fixing (acetylene reducing) activity in a cut upland sward. Grass Forage Sci 43, 253-262

Mouat $\mathrm{MCH}$, Walker TW (1959) Competition for nutrients between grasses and white clover. 1. Effect of grass species and nitrogen supply. Plant Soil $11,30-40$ 
Mouat MCH (1983) Competitive adaptation by plants to nutrient shortage through modification of root growth and surface charge. $N Z$ J Agric Res 26, 327-332

Sackville-Hamilton NR (1994) Replacement and additive designs for plant competition studies. $J$ Appl Ecol 31, 599-603

Salette J, Lemaire G (1981) Sur la variation de la teneur en azote de graminées fourragères pendant leur croissance : formulation d'une loi de dilution. C R Séances Acad Sci 292, 848-855

Sinoquet H, Moulia B, Gastal F, Bonhomme R, VarletGrancher C (1990) Modelling the radiative balance of the components of a binary mixed canopy: application to a white clover/fall fescue mixture. Acta CEcologica 11, 469-486

Solangaarachchi SM, Harper JL (1987) The effect of canopy filtered light on the growth of white clover (Trifolium repens $\mathrm{L}$ ). CEcologia 72, 372-376

Soussana JF, Arregui C, Hazard L (1989) Assimilation du nitrate et fixation symbiotique dans les associations trèfle blanc - ray-grass anglais. In : Proc of the XVI Int Grassl Cong, AFPF, Nice, France, 147148
Soussana JF, Vertès F, Arregui MC (1995a) The regulation of clover shoot growing points density and morphology during clover decline in mixed swards. Eur J Agron (sous presse)

Soussana JF, Faurie O, Sinoquet H (1995b) Radiation and nitrogen acquisition and use in simulated grassclover swards. In : Ecophysiology of Tropical Intercropping (Sinoquet $\mathrm{H}, \mathrm{Cruz} \mathrm{P}$, eds), INRA, Paris, 331-341

Streeter $J(1988)$ Inhibition of legume nodule formation and $\mathrm{N}_{2}$ fixation by nitrate. CRC Crit Rev Plant Sci 7 , $1-23$

Vallis I, Haydcock KP, Ross PJ, Henzell EF (1967) Isotopic studies of the uptake of small additions of $15 \mathrm{~N}$ by Rhodes grass and Townsville lucerne. Aust $J$ Agric Res 18, 865-877

Varlet-Grancher C, Moulia B, Jacques R (1989) Phytochrome mediated effects on white clover morphogenesis. In : Proc of the XVI Int Grassl Cong, AFPF, Nice, France, 477-478

Wit CT de, Tow PG, Ennick GC (1966) Competition between legumes and grasses. Verslagen van Landbouwkundige Onderzoekingen 687, 1-30 